\title{
Enhanced performance of microbial fuel cells with electron mediators from
}

\section{anthraquinone/polyphenols-abundant herbal plants}

Tao Li†, Xiao-Li Yang*, Qiao-Ling Chen†, Hai-Liang Song**, + Zhen He§, Yu-Li Yang $\ddagger$

$\uparrow$ School of Civil Engineering, Southeast University, Nanjing 211189, China

$\$$ School of Environment, Nanjing Normal University, Jiangsu Engineering Lab of Water and

Soil Eco-Remediation, Nanjing 210023, China

$\S$ Department of Energy, Environmental and Chemical Engineering, Washington University

in St. Louis, MO 63130, USA

* Correspondence to: X.-L. Yang, yangxiaoli@seu.edu.cn (X.-L. Yang)

**Correspondence to: H.-L. Song, hlsong@njnu.edu.cn (H.-L. Song)

Number of pages: 22

Number of figures: 4

Number of tables: 3

Section S1: A multiple color staining technique and confocal laser scanning microscopy (CLSM) analysis

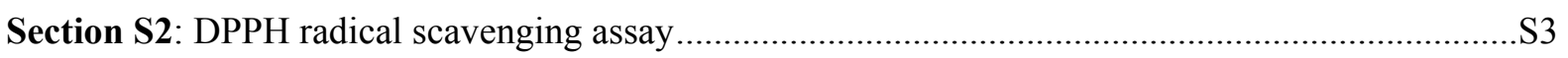

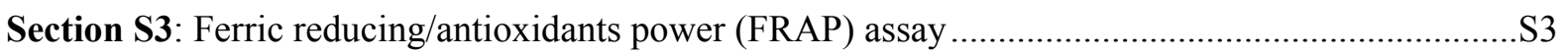

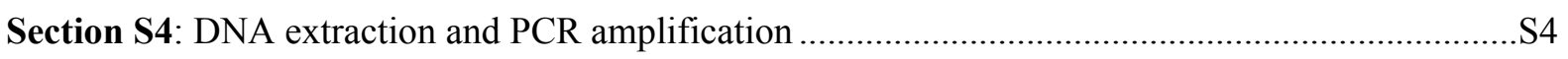

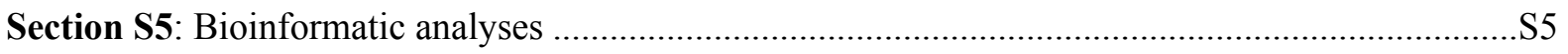

Section S6: Assessment on redox ability of six herbal plants.......................................................... 5

Section S7: Pretreatment methods selection of herbal plants ........................................................... 9

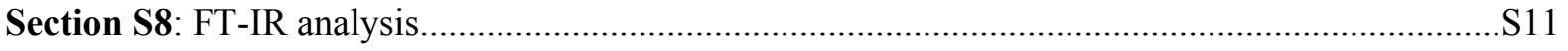

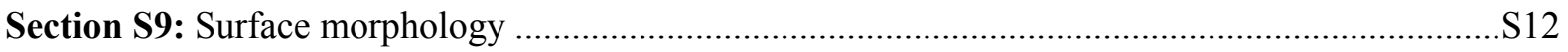

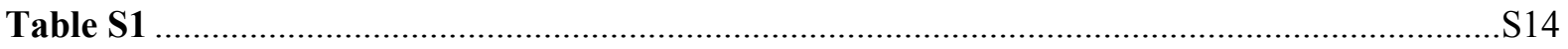

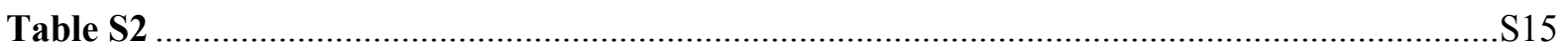

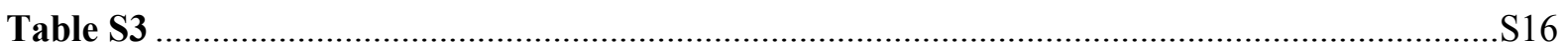

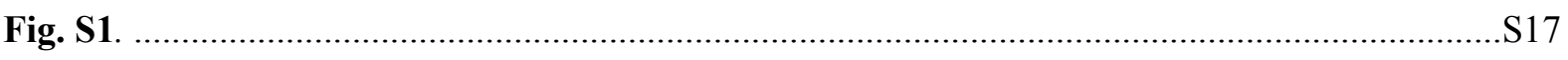

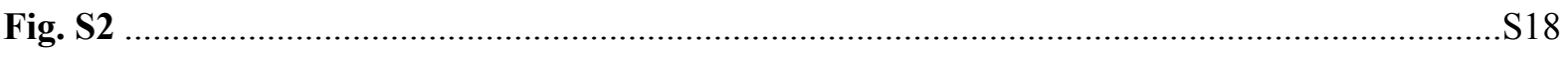

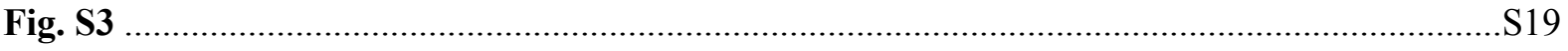

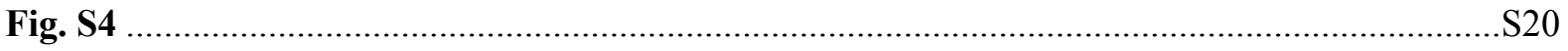

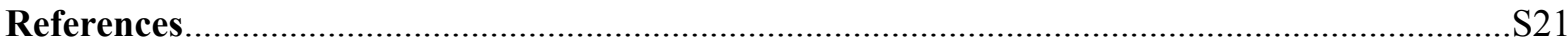




\section{Supporting Information}

This supporting information has 22 pages, including 9 associated text sections (namely Section S1, Section S2, Section S3, Section S4, Section S5, Section S6, Section S7, Section S8, Section S9), 3 Tables (Table S1, Table S2, Table S3), 4 Figures (Fig. S1, Fig. S2, Fig. S3, Fig. S4).

Section S1: A multiple color staining technique and confocal laser scanning microscopy (CLSM) analysis

After more than 120 days of exposure to the herbal plants EMs, the suspended sludge and biofilm were collected by filtering $5 \mathrm{~mL}$ mixed liquor through a $0.45 \mu \mathrm{m}$ PES (polyether sulfone) filter. Then the samples were transferred onto a semi-solid agar surface through inverting the filter membrane onto the agarose matrix to avoid entire dehydration. ${ }^{1}$ The agarose matrix was pre-spread evenly onto a microscopic slide well with a polished spherical depression. The filter was dried at room temperature for $10 \mathrm{~min}$ and then carefully removed using a forcep. ${ }^{2}$ For biofilm selection, the well-sized biofilm samples were taken from anode carbon brush and placed on slides. All samples were stored in $4{ }^{\circ} \mathrm{C}$ refrigerator before staining. Before multiple fluorescent staining, all samples were fixed with $2.5 \%$ glutaraldehyde for 12 h, followed by staining with fluorescein isothiocyanate (FITC) (Aladdin, China), concanavalin A (Con A) (Aladdin, China), and calcofluor white (CW) (Sigma, St.Louis, MO) to target protein (PRO), $\alpha$-, and $\beta$-D-glucopyranose polysaccharide (PS), respectively. ${ }^{3}$ After $1 \mathrm{M}$ sodium bicarbonate $\left(\mathrm{NaHCO}_{3}\right)$ buffer was used for keeping the amine group in a nonprotonated form, ${ }^{4} 20 \mu \mathrm{L}$ FITC ( $5 \mathrm{~g} / \mathrm{L}$, dissolved in dimethylsulfoxide) was first dripped onto the sample for $1 \mathrm{~h}$. Thereafter, $100 \mu \mathrm{L} 0.25 \mathrm{~g} / \mathrm{L}$ Con A was dropped on the samples for $30 \mathrm{~min}$. 
Next, $200 \mu \mathrm{L} 0.5 \mathrm{~g} / \mathrm{L} \mathrm{CW}$ was added on the samples for another $30 \mathrm{~min}$. After the samples were stained, PBS was dropped on the slides to wash the samples two times to remove excess stain. Finally, the stained samples were characterized by CLSM (ZEISS LSM 710, ZEISS, Germany). The excitation/emission wavelengths for observing FITC, Con A and CW are 488 $\mathrm{nm} / 500-540 \mathrm{~nm}$ (green), $552 \mathrm{~nm} / 550-600 \mathrm{~nm}$ (red) and $405 \mathrm{~nm} / 410-480 \mathrm{~nm}$ (blue).

In addition, the MATLAB ${ }^{\circledR}$ program was applied to convert the CLSM images to gray images with pixel values between 0 (black) and 255 (white) according to the literature reported ${ }^{5}$ and the pixel value of each point $(807 \times 807)$ was subsequently extracted and used for analysis.

\section{Section S2: DPPH radical scavenging assay}

$0.5 \mathrm{~g}$ herbal plants were powdered and then dissolved in $50 \mathrm{~mL} 50 \%(\mathrm{~V} / \mathrm{V})$ ethanol for 45 min extraction by ultrasonic at $45^{\circ} \mathrm{C}$. Then, transfer extracting solution into a $50 \mathrm{~mL}$ volumetric flask and diluted the solution to a final volume of $50 \mathrm{~mL}$. The sample solution was prepared for using freshly. Dissolve $1 \mathrm{mg}$ DPPH in $24 \mathrm{~mL}$ absolute ethanol to configure the mother liquor. Then $0,40,80,120,160,200 \mu \mathrm{L}$ sample solution and $1000,960,920,880,840,800 \mu \mathrm{L}$ absolute ethanol were added into $2 \mathrm{~mL}$ bulk liquor and ensure final volume $3 \mathrm{~mL}$. After $30 \mathrm{~min}$ incubation period at room temperature in the darkness, the decrease in the absorbance (Abs) was measured at $520 \mathrm{~nm}$. The percentage of inhibition was calculated according to the following equation: DPPH free radical scavenging rate $=\frac{A b s(\operatorname{Control})-A b s(\text { Sample })}{A b s(\text { Control })} \times 100 \%$. Inhibitory activities of different concentrations of the isolated compounds were assessed to determine the half maximal inhibitory concentration $\left(\mathrm{IC}_{50}\right) \cdot{ }^{6}$ All tests were carried out in triplicate.

\section{Section S3: Ferric reducing/antioxidants power (FRAP) assay}


First, the standard curve of concentration and absorbance was made. Then, the FRAP reagent was prepared freshly. The stock solution included $25 \mathrm{~mL}$ acetate buffer (300 mM, pH 3.6), 2.5 mL $10 \mathrm{mM}$ TPTZ (2,4,6-tripyridyl-s-triazine) solution in $40 \mathrm{mM} \mathrm{HCl}$ and $2.5 \mathrm{~mL}$ of $20 \mathrm{mM}$ $\mathrm{FeCl}_{3} \cdot 6 \mathrm{H}_{2} \mathrm{O}$. The mixture was warmed at $37^{\circ} \mathrm{C}$ before using. $18 \mathrm{~mL}$ FRAP solution and $2 \mathrm{~mL}$ sample solution were put into the $25 \mathrm{~mL}$ colorimetric tube and mixed ( 3 parallel samples). Then, the mixed liquor was heated in water bath at $37^{\circ} \mathrm{C}$ for $10 \mathrm{~min}$. The absorbance was measured at $593 \mathrm{~nm}$ after $30 \mathrm{~min}$. Aqueous solutions of herbal plants extract concentration was between 0 and $300 \mu \mathrm{M}$ for calibration. Tests were carried out in triplicate.

\section{Section S4: DNA Extraction and PCR Amplification}

Purity and quality of the genomic DNA were checked on $0.8 \%$ agarose gels. The V3-4 hypervariable region of bacterial $16 \mathrm{~S}$ rRNA gene were amplified with the primers $338 \mathrm{~F}$ (ACTCCTACGGGAGGCAGCAG) and 806R (GGACTACHVGGGTWTCTAAT) ${ }^{7}$ by PCR $\left(95^{\circ} \mathrm{C}\right.$ for $5 \mathrm{~min}$, followed by 32 cycles of $95{ }^{\circ} \mathrm{C}$ for $45 \mathrm{~s}, 55^{\circ} \mathrm{C}$ for $50 \mathrm{~s}$ and $72{ }^{\circ} \mathrm{C}$ for $45 \mathrm{~s}$ with a final extension at $72{ }^{\circ} \mathrm{C}$ for $10 \mathrm{~min}$ ). PCR was carried out on a Mastercycler Gradient (Eppendorf, Germany) using $25 \mu \mathrm{L}$ reaction volumes, containing $12.5 \mu \mathrm{L} 2 \times$ Taq PCR MasterMix, $3 \mu \mathrm{L}$ BSA (2 ng/ $\mu \mathrm{L}), 2$ Primer $(5 \mu \mathrm{M}), 2 \mu \mathrm{L}$ template DNA, and $5.5 \mu \mathrm{L} \mathrm{ddH}_{2} \mathrm{O}$. Three PCR products per sample were pooled to mitigate reaction-level PCR biases. The PCR products were purified using a QIAquick Gel Extraction Kit (QIAGEN, Germany), quantified using Real Time PCR, and sequenced at Allwegene Company, Beijing. 


\section{Section S5: Bioinformatic Analyses}

The raw data were first screened and sequences were removed from consideration if they were shorter than $200 \mathrm{bp}$, had a low-quality score $(\leq 20)$, contained ambiguous bases or did not exactly match to primer sequences and barcode tags. Qualified reads were separated using the sample-specific barcode sequences and trimmed with Illumina Analysis Pipeline Version 2.6. And then the dataset was analyzed by QIIME. The sequences were clustered into operational taxonomic units (OTUs) at a similarity level of $97 \%,{ }^{8}$ to generate rarefaction curves and to calculate the richness and diversity indices. The Ribosomal Database Project (RDP) Classifier tool was used to classify all sequences into different taxonomic groups. ${ }^{9}$

To examine the similarity between different samples, clustering analyses and PCA were used based on the OTU information from each sample using R. ${ }^{10}$ The evolution distances between microbial communities from each sample were calculated using the tayc coefficient and represented as an Unweighted Pair Group Method with Arithmetic Mean (UPGMA) clustering tree describing the dissimilarity (1-similarity) between multiple samples. ${ }^{11}$ A Newickformatted tree file was generated through this analysis. To compare the membership and structure of communities in different samples, heat maps were generated with the top 20 OTUs using Mothur. ${ }^{12}$

\section{Section S6: Assessment on redox ability of six herbal plants}

Cyclic voltammetry (CV) technology was widely applied in the evaluation of electrochemically active substance and antioxidant capacity containing in plants. ${ }^{13}$ Upon the electrochemistry parameter obtained in CV curves (e.g., redox peak potential, $E p_{a} \& E p_{c}$ and redox peak current, $\left.I \mathrm{p}_{\mathrm{a}} \& \mathrm{I} \mathrm{p}_{\mathrm{c}}\right)$, redox reversibility and redox activity can be inferred. In actual 
research results, if there is an only oxidative peak or reductive peak existing in CV curves, it means that the substance was oxidized or reduced irreversibly. As a kind of catalyst which can catalyze microbial electron transfer, it is necessary for electron mediators (EMs) to achieve stable redox reversibility. Simultaneous oxidation peaks and reduction peaks in CV would determine the potential feasibility acting as EMs. As Fig. S1(a-f) revealed, six herbal plants had one single oxidation peak (Fig. S1(c) and Fig. S1(d)) or both two redox peaks (Fig. S1(a), 1(b), 1(e), 1(f)). The water leaching liquid of H.reynoutria (Fig. S1(c)) and E. aloe (Fig. S1(d)) showed oxidation peak at $235 \mathrm{mV}$ and $311 \mathrm{mV}$, but corresponding reduction peaks were hardly visible, possibly due to the main detected electroactive substances contained in the two plants were oxidized irreversibly under micro-voltage condition. The CV curves of B.rheum (Fig. S1(a)) and T.fallopia (Fig. S1(b)) water leaching liquid showed a pair of approximately symmetric redox peaks. Meanwhile, two and three pairs of redox peaks were observed in L.Rubia (Fig.S1(e)) and Catsia tora Linn (Fig. S1(f)) group, respectively. That means, 2 and 3 main redox-active substances exist in the two plants. According to the CV analysis above, the four herbal plants (B.rheum, T.fallopia, L. Rubia, and Catsia tora Linn) were possibly potential EMs contributing from their special redox characteristics and quasi-reversibility of redox processes. ${ }^{14}$ The electrochemical parameters for these cyclic voltammograms are given in Table S1.

The ratio of $\operatorname{Ip}_{\mathrm{a}}$ (anode peak current) to $\mathrm{Ip}_{\mathrm{c}}$ (cathodic peak current) can be used to determine the degree of reversibility. $\mathrm{Ip}_{\mathrm{a}} / \mathrm{Ip}_{\mathrm{c}}$ approaches 1.0 for a fully reversible process. ${ }^{15}$ It can be seen from Table S1 that B.rheum, T.fallopia and L. Rubia redox peak current ratio were close to 1.0. The peak current ratio of Catsia tora Linn peak 1 was more than 2.0 and the peak current ratio 
of peak $2 \& 3$ were less than 0.2 . The consequence demonstrated that the electrochemical active substance contained in B.rheum, T.fallopia and L. Rubia may have stronger reversibility than Catsia tora Linn.

The redox peak current value in CV curves was positively correlated with the concentration of substance under certain conditions. ${ }^{16}$ According to the reports conducted in food chemistry and pharmacology, the electrochemical active substances contained in polygonaceae and rubiaceae plants were represented by anthraquinones with phenol hydroxyl. Hence, the certain redox active compounds content for the corresponding peak of four herbal plants ranked: Catsia tora Linn $($ peak 1$)>$ T.fallopia $>$ L.Rubia $($ peak 1$)=$ Catsia tora Linn $($ peak 2$)>$ B.rheum $>$ Catsia tora Linn (peak 3) $>$ L.Rubia (peak 2). However, the extract concentrations should be above threshold levels to significantly exhibit reductive or/and oxidative potential peaks in $\mathrm{CV}$ profiles. ${ }^{17}$ Therefore, the ranking of redox-active compounds content needs to be verified further by other methods afterward.

The difference of redox peak potential in cyclic voltammetry curve was expressed by formula (1):

$$
\Delta \mathrm{E}=E_{p a}-E_{p c}
$$

According to the concept from classical polarography, determination of the half-wave potential was calculated by the formula:

$$
E_{1 / 2}=E_{p a}-\frac{1}{2} \Delta \mathrm{E}=\left(E_{p a}+E_{p c}\right) / 2
$$

$\mathrm{E}_{1 / 2}$ was associated with thermodynamic formal redox potential:

$$
E_{1 / 2}=E^{0}+(R T / n F) \ln \left(\mathrm{D}_{R} / \mathrm{D}_{0}\right)^{\frac{1}{2}}
$$

where $\mathrm{n}$ denotes the number of electrons appearing in a redox reaction, $\mathrm{F}$ is Faraday's constant 
$(96,485 \mathrm{C} / \mathrm{mol}), \mathrm{R}$ is universal gas constant $(8.314 \mathrm{~J} / \mathrm{mol} \bullet \mathrm{K}), \mathrm{T}$ is absolute temperature $(\mathrm{K})$ and $D_{R}$ and $D_{0}$ is diffusion coefficient of oxidized and reduced forms $\left(\mathrm{cm}^{2} / \mathrm{s}\right)$. In general, there is little difference between $\mathrm{D}_{\mathrm{R}}$ and $\mathrm{D}_{0}$, so $\mathrm{E}_{1 / 2}$ value approximatively equal to $\mathrm{E}^{0}$. Upon Nernst law, for a quasi-reversible process, $\Delta \mathrm{E}$ was given by:

$$
\Delta \mathrm{E}=\frac{2.3 R T}{n F}=\frac{59.0}{n} \mathrm{mV}
$$

Formula (4) can be used to calculate the electron numbers transferred in a quasi-reversibility redox reaction. The $n$ value for B.rheum, T.fallopia, L.Rubia and Catsia tora Linn was calculated to be $0.73,0.58,0.39$ (L. Rubia peak 1), 0.29 (L. Rubia peak 2), 0.69 (Catsia tora Linn peak 1), 0.56 (Catsia tora Linn peak 2) and 0.72 (Catsia tora Linn peak 3). Most of the electron numbers were around 1 and results revealed that the reactions happened in aqueous solution for four EMs candidates were likely to be single electron transfer reactions.

To verify whether the occurrence of redox peaks in plant water extracts is caused by anthraquinones, the CVs for four pure anthraquinones convinced in the herbal plants before were shown in Fig. S2. The position of pure substance redox peaks was similar as herbal plants water leach liquor. Compared to pure chemical compounds (Rhein $(\mathrm{n} \approx 0.77)$, Aloe-emodine $(\mathrm{n} \approx 3.47)$, Alizarin $\left.\left(\mathrm{n}_{1} \approx 0.86, \mathrm{n}_{2} \approx 1.47, \mathrm{n}_{3} \approx 1.40\right)\right)$, the herbal plants' water leaching liquid demonstrated similar number of electron transfer (except Aloe-emodine) and moderate electron-shuttling capabilities. The possible reasons were that some non-EM-associated mixing impurities in herbal plants (e.g., some essential oils, fatty acids and plant sterols) were oxidized irreversibly and adsorbed on the electrode surface. This behavior altered electrochemistry response of electrode much and decreased the peak current. ${ }^{16}$ This provided a basis for subsequent reaction mechanism analysis. 
The electrode reaction process is generally controlled by two steps: diffusion process and adsorption process (kinetic control). The electroactive compounds reach the surface of the electrode through a diffusion process between the solution and the electrode first and then adsorbed on the surface of the electrode through adsorption process to participate in the redox reaction. Between the two consecutive processes, the reaction was controlled by the slower one. The reaction controlling step was determined upon the relationship between peak current and sweep speed in CV curves. Our research results indicated that the peak current of the four herbal plants was proportional to sweep speed, that meant the plants' water leaching liquid electrode processes were controlled by diffusion.

\section{Section S7: Pretreatment methods selection of herbal plants}

Furthermore, the CV results conducted in Section S6 demonstrated that the electrochemical performance of alternative herbal plants by water immersion pretreatment was not satisfactory. Hence, the acid pretreatment and ultrasonic pretreatment were applied to alternative herbal plants for enhancing the electrochemistry active compounds releasing. Fig. 2 showed the cyclic voltammograms of herbal plants under different pretreatment methods.

Through the figure, we can see that the same pretreatment method had different effects on the electrochemical activity of different plants. And different pretreatment methods also influenced the performance of the same herbal plants much. For acid pretreatment, an interesting phenomenon was observed that the lower concentration acid (2\%) promoted the redox peak current more than that of higher concentration (10\% and 30\%) for B.rheum, L.Rubia and Catsia tora Linn, while for T.fallopia, 30\% acid achieved the highest peak current improvement. Ultrasonic pretreatment experiment results indicated that ultrasonic treatment 
just improved the electrochemistry performance of B.rheum and T.fallopia a little, but it had few effects on Catsia tora Linn and L.Rubia. What's more, the response of different plant samples to ultrasonic frequencies varies greatly. For B.rheum, high ultrasonic frequency (70 $\mathrm{kHz}$ ) can promote the electrochemical response effectively. But the lower ultrasonic frequency $(30 \mathrm{kHz})$ achieved better peak current improvement for T.fallopia. In general, the area of CV curves can reflect the total amount of electron transfer during the whole electrochemical process (forward scan and reverse scan) approximately. Moreover, the electron transfer number has been considered as a significant symbol of quantifying electrochemical capabilities. The areas of closed CV loops can be expressed by the equation as follows:

$$
S=\int_{V_{\text {Low }}}^{V_{H i g h}}\left(I_{\text {High }}-I_{\text {Low }}\right) d V
$$

where $\mathrm{S}$ represents the $\mathrm{CV}$ curves area; $V_{\text {High }}$ and $V_{\text {Low }}$ represent the initial scan voltage and end scan voltage; $I_{H i g h}$ and $I_{\text {Low }}$ represent the highest and lowest current during the whole scan process, respectively. The results showed in Fig. 2(a-d) revealed that the areas of CV loops for four plants treated by acid were larger than those treated by ultrasonic. And the peak current improvement proportion of acid treatment was greater than ultrasonic treatment. This indicated that acid treatment was more effective than ultrasonic in improving plants electrochemical activity.

For B.rheum, T.fallopia and Catsia tora Linn, both pretreatment methods enhanced the electrochemical activity in the form of peak current at the potential $\left(\mathrm{Ep}_{\mathrm{a}} \& \mathrm{Ep}_{\mathrm{c}}\right)$ 200-400 $\mathrm{mV}$. However, an amazing result was observed in L.Rubia group that two new peaks appeared at $300-400 \mathrm{mV}$ and $600-700 \mathrm{mV}$ after $2 \%$ acid treatment, which indicated that two new electroactive substances released and the electron mediation potential of L.Rubia was 
strengthened.

To illuminate the changes of herbal plants before and after pretreatment more clearly, FT-IR and SEM technology were employed afterward.

\section{Section S8: FT-IR analysis}

Four untreated, acid $(2 \%)$ and ultrasonic $(72 \mathrm{kHz})$ pretreatment herbal plants materials were analyzed by FT-IR. The FT-IR spectra of four plants was shown in Fig. S3. Through Fig. S3(a), it can be seen that four herbal plants displayed similar absorption peaks at 1030, 1180, 1470, $3270 \mathrm{~cm}^{-1}$ corresponding to $-\mathrm{C}-\mathrm{O}-\mathrm{C}$ asymmetric stretching vibration, -C-O-C symmetrical stretching vibration, $-\mathrm{CH}_{2}$ scissoring bend and $-\mathrm{OH}$ stretch vibration ${ }^{18}$, which were the cellulose characteristic peak before pretreatment. Meanwhile, all herbal plants showed absorption peaks with different intensity at 1600,1500 and $1450 \mathrm{~cm}^{-1}$ which confirmed the existence of aromatic compounds. The results mentioned above proved the polyphenol substance was contained in the selected plants and this was consistent with the previous research. ${ }^{19}$ However, some apparent variations also existed between four alternative herbal plants. L. Rubia displayed distinct absorption peaks at 779 and $1313 \mathrm{~cm}^{-1}$ corresponding to $\mathrm{C}-\mathrm{H}$ external bending vibration and CO telescopic which exhibited the differences of number and position of hydroxy substituents on the benzene ring compared with other herbal plants. Catsia tora Linn displayed distinct absorption peaks at $1537 \mathrm{~cm}^{-1}(\mathrm{C}=\mathrm{C}$ skeletal vibration $), 1742 \mathrm{~cm}^{-1}(\mathrm{C}=\mathrm{O}$ telescopic vibration $)$, 2852 and $2920 \mathrm{~cm}^{-1}$ (C-H stretching vibration). This disparity may influent the pretreatment performance. After acid and ultrasound pretreatment, Fig. S3(b), Fig. S3(c), Fig. S3(e) showed that B.rheum, T.fallopia and L.Rubia obtained obvious absorption peak at around $1738 \mathrm{~cm}^{-1}$ compared with the control groups. According to the FT-IR database, the wavenumber 
represented $\mathrm{C}=\mathrm{O}$ stretching vibration, which was the characteristic peak of six/seven-membered cycloketone. It was consistent with anthraquinone compound structures showed in Table S2. For Catsia tora Linn, Fig. S3(d) displayed a new absorption peak appeared at $1452 \mathrm{~cm}^{-1}$ after pretreatment which was frequently found in the skeletal vibration of substituted benzenes. Furthermore, B.rheum also showed an obvious absorption peak at $1146 \mathrm{~cm}^{-1}$, which indicated that C-O stretching vibration and this was frequently observed in tertiary alcohols. All FT-IR results of herbal plants with pretreatment showed the appearance of anthraquinones representative groups, which was due to the increasement of anthraquinones. What's more, acid pretreatment achieved higher peak intensity than ultrasonication. The results agreed with the $\mathrm{CV}$ analysis that $2 \%$ acid pretreatment was more favorable than ultrasonication for anthraquinones releasing.

\section{Section S9: Surface morphology}

Surface areas and surface roughness might affect the release of electroactive compounds and the adhesion of microorganism. SEM was employed to reveal the surface morphology changes before and after acid (2\%) and ultrasound $(72 \mathrm{kHz})$ pretreatment. The results were shown in Fig. S4. It can be seen that different pretreatment methods caused distinct surface morphology changes between four selected herbal plants. Before pretreatment, the surface of B.rheum, Catsia tora Linn, L.Rubia was smooth and few porous and concave-convex structures were found, while the surface of T. fallopia was rough and some microsphere structure and fiber structure were closely cross-linked. After ultrasonic pretreatment, different degrees of fragmentation and changes mainly happened on superficial layer structures of the herbal plants. Compared with the ultrasonic group, after acid pretreatment, the surface morphology of four 
plants changed almost completely and large numbers of pore broken structures occurred. The increased rough stratified structure can provide more space for microorganism adhesion. Furthermore, a crucial finding upon the SEM images was that ultrasonic treatment only damaged the surface structure-broke the surface and expose the inner; while the acid treatment contributed to unfolding inner cross-linked structure and destroying the covering layer consisting of waxes and pectin-like substances on the surface of cellulose. This difference between the two pretreatment methods played an important role in electrochemical active substances release. Surface morphology analysis corresponded to the electrochemistry analysis that $2 \%$ acid pretreatment was more suitable for the release of electrochemical active substances. 
Table S1 Cyclic voltammetry peak parameters of B.rheum, T.fallopia, L.Rubia and Catsia tora Linn, treated for $24 \mathrm{~h}$ in $200 \mathrm{~mL}$ water at $28^{\circ} \mathrm{C}$

\begin{tabular}{ccccccccc}
\hline \multirow{2}{*}{ Name } & $\begin{array}{c}\text { Peak } \\
\text { number }\end{array}$ & $\mathrm{Ep}_{\mathrm{a}}(\mathrm{mV})$ & $\mathrm{Ep}_{\mathrm{c}}(\mathrm{mV})$ & $\mathrm{Ip}_{\mathrm{a}}(\mu \mathrm{A})$ & $\mathrm{Ip}_{\mathrm{c}}(\mu \mathrm{A})$ & $\Delta \mathrm{E}(\mathrm{mV})$ & $\mathrm{Ip}_{\mathrm{a}} / \mathrm{Ip}_{\mathrm{c}}$ & $\mathrm{E}^{0}(\mathrm{mV})$ \\
\hline B.rheum & 1 & 274 & 193 & 0.167 & 0.194 & 81 & 0.86 & 233.5 \\
T.fallopia & 1 & 274 & 172 & 0.218 & 0.416 & 102 & 0.52 & 223 \\
L. Rubia & 1 & 245 & 92 & 0.212 & 0.29 & 153 & 0.73 & 173 \\
Catsia tora & 2 & -558 & -356 & 0.127 & 0.268 & 202 & 0.47 & -457 \\
Linn & 1 & 443 & 357 & 0.661 & 0.183 & 86 & 3.61 & 400 \\
& 2 & -39 & -145 & 0.212 & 1.238 & 106 & 0.17 & -92 \\
\hline
\end{tabular}


Table S2 Structures of the anthraquinone contained in herbal plants used in this study

No..


Table S3 Richness and evenness of microbial community in MFC biofilm

\begin{tabular}{cccccc}
\hline Sample ID & Chao 1 & $\begin{array}{c}\text { Observed } \\
\text { species }\end{array}$ & PD whole tree & Shannon & OTUs \\
\hline $\mathrm{MFC}_{0 \_} \mathrm{B}$ & 1073.7063 & 834.6 & 70.33678 & 4.99464 & 861 \\
$\mathrm{MFC}_{1} \mathrm{~B}$ & 1192.31199 & 981.7 & 81.09684 & 4.01482 & 1010 \\
$\mathrm{MFC}_{2} \mathrm{~B}$ & 1108.26168 & 888.3 & 74.75114 & 4.56295 & 917 \\
$\mathrm{MFC}_{3} \mathrm{~B}$ & 1160.73357 & 868.5 & 73.97296 & 4.50076 & 897 \\
$\mathrm{MFC}_{4} \mathrm{~B}$ & 1395.52072 & 1193.9 & 107.019 & 5.82581 & 1224 \\
$\mathrm{MFC}_{5} \mathrm{~B}$ & 839.83302 & 607.6 & 56.33088 & 2.927 & 628 \\
$\mathrm{MFC}_{6 \_} \mathrm{B}$ & 1178.49394 & 966.6 & 89.99703 & 4.85244 & 994 \\
\hline
\end{tabular}



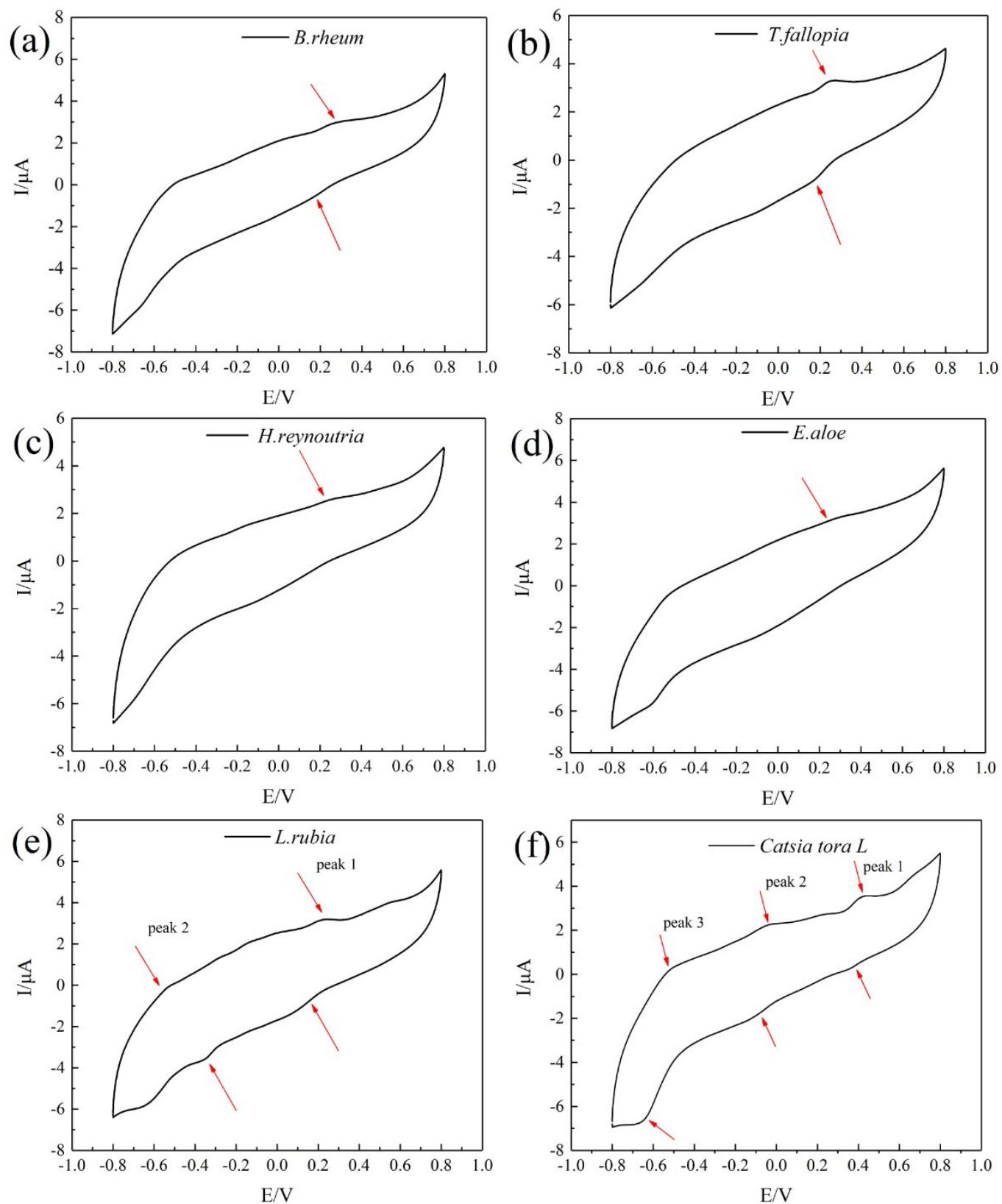

Fig. S1 Cyclic voltammogram of six herbal plants (water soaking; $50 \mathrm{mV}$ scanning speed): (a) B.rheum, (b) T.fallopia, (c) H.reynoutria, (d) E.aloe, (e) L.Rubia, (f) Catsia tora Linn. 

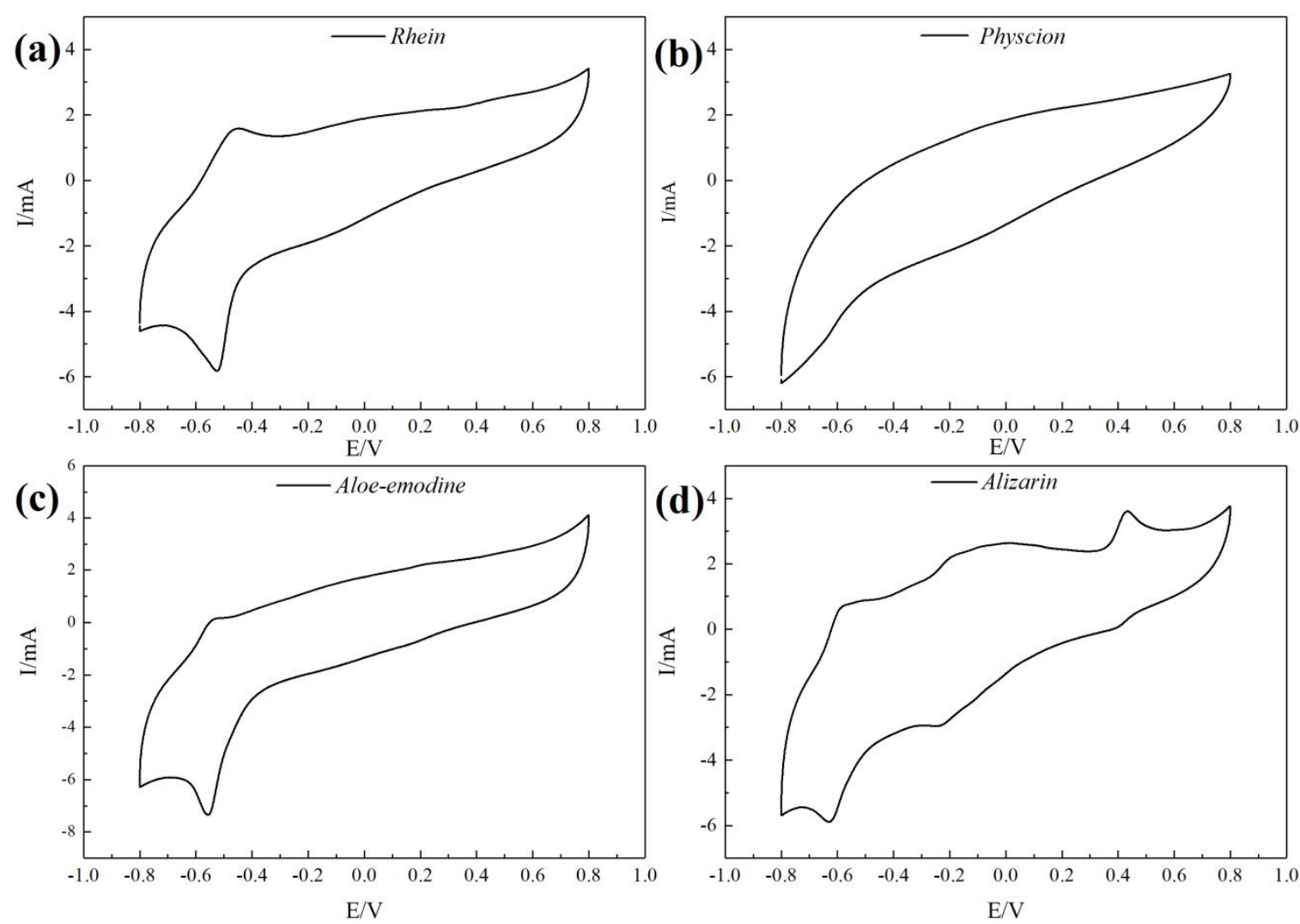

Fig. S2 Cyclic voltammogram of representative pure substances contained in six herbal plants $(50 \mathrm{mV}$ scanning speed) (a) Rhein; (b) Physcion; (c) Aloe-emodine; (d) Alizarin 
(a)

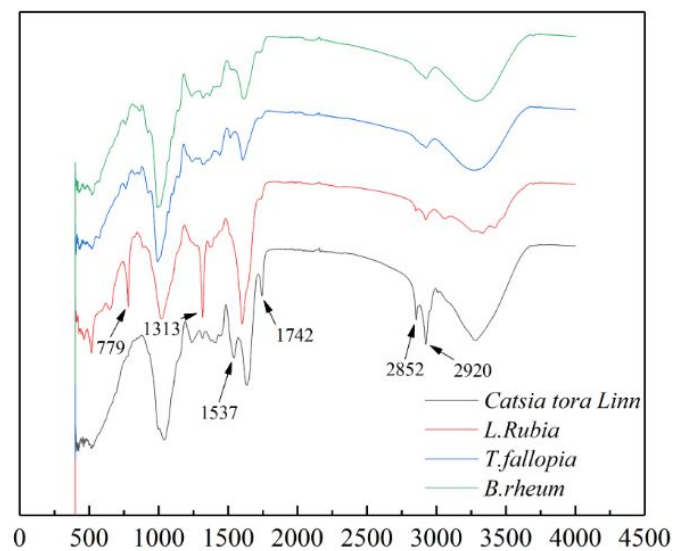

Wavenumbers $/ \mathrm{cm}^{-1}$
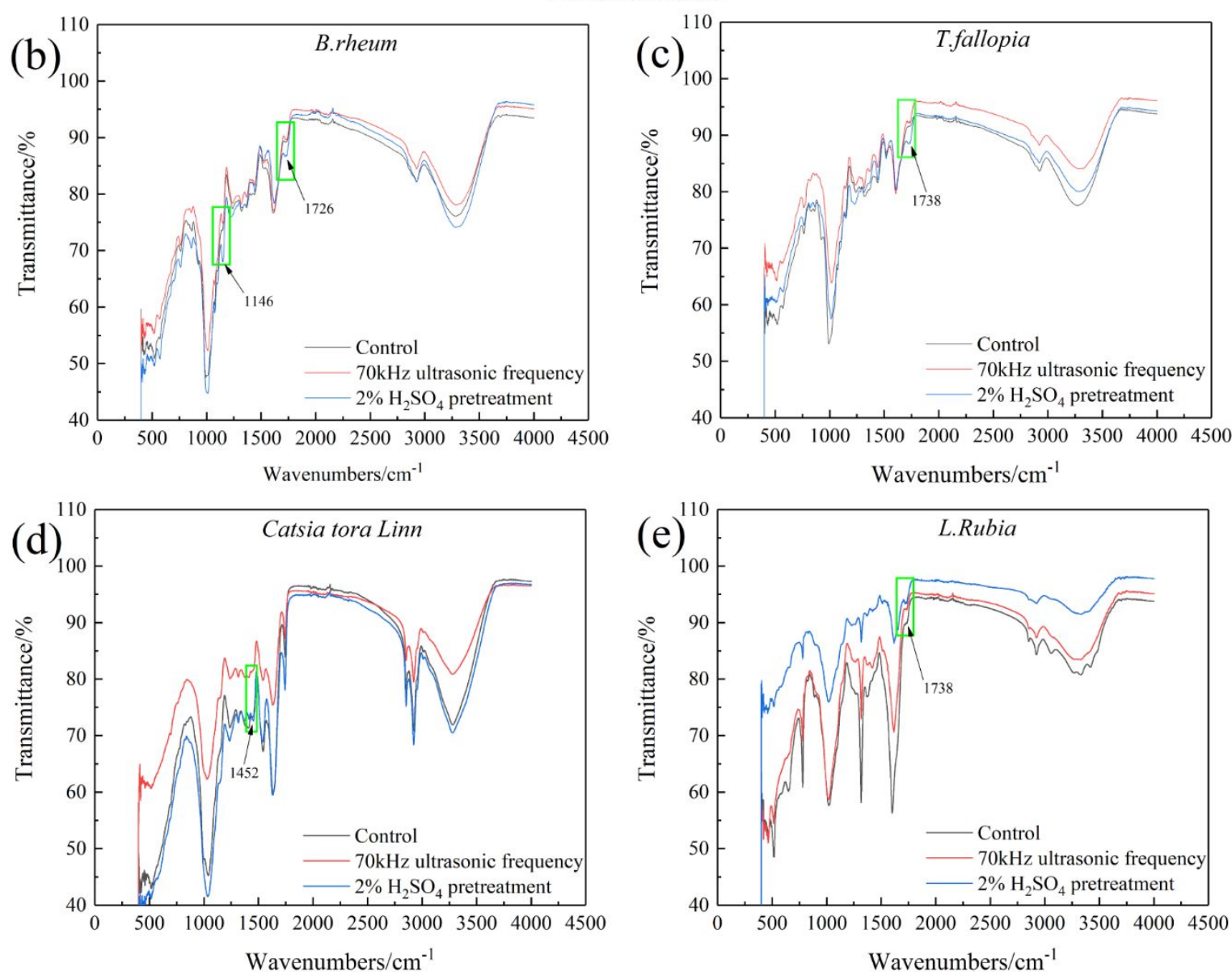

Fig. S3 FT-IR spectra of untreated (a) and acid/ ultrasound pretreatment herbal plants (b)-(e). 


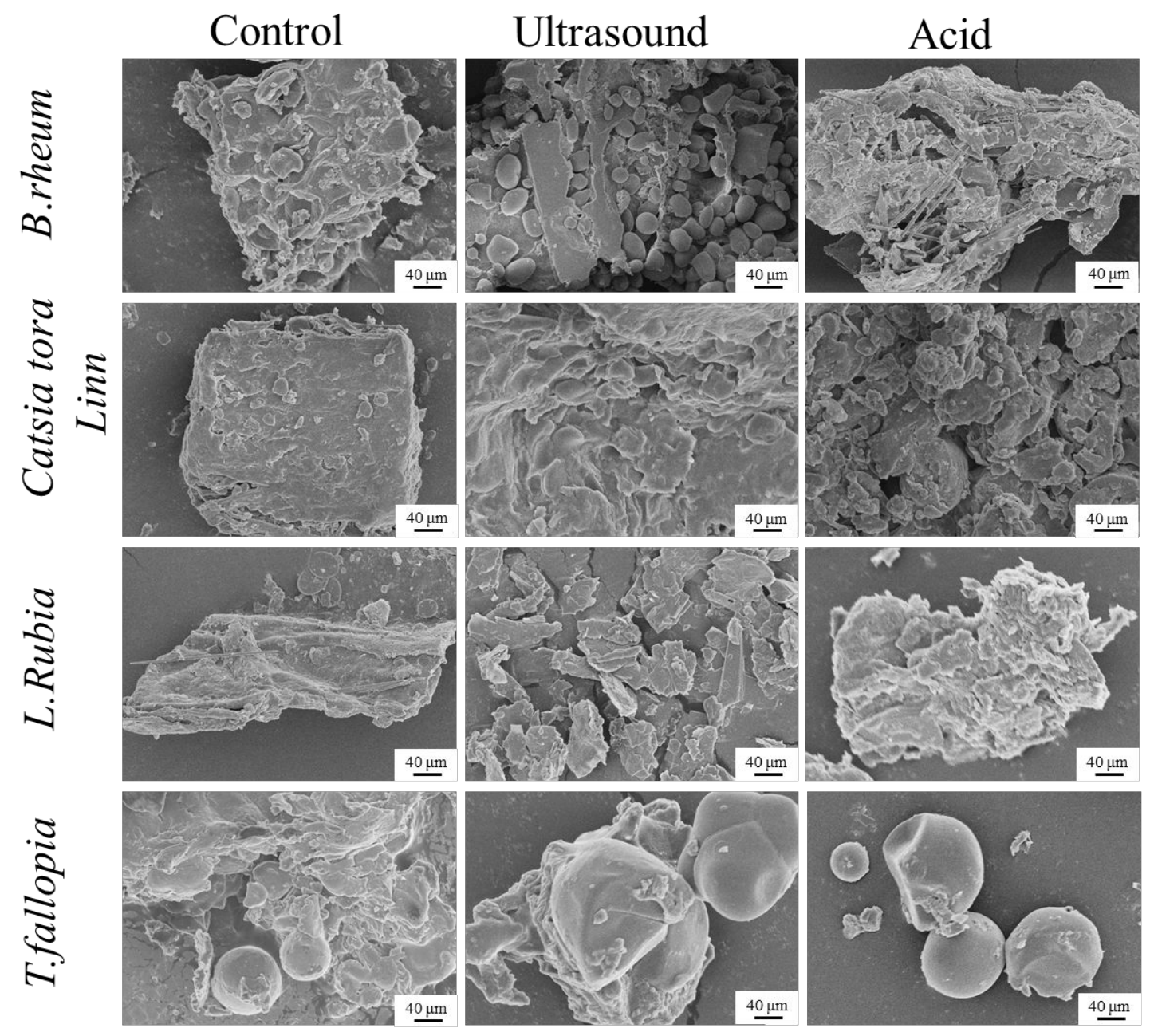

Fig. S4 SEM images of herbal plants before and after the pretreatment $(\times 2500)$ 


\section{References:}

(1) Basuvaraj, M.; Fein, J.; Liss, S.N. Protein and polysaccharide content of tightly and loosely bound extracellular polymeric substances and the development of a granular activated sludge floc. Water Res 2015, 82, 104-117.

(2) You, G.; Wang, P.F; Hou. J,; Wang, C.; Qian, .J.; Ao. Y.H.; Chen, J.; Miao, L.Z.; Xu. Y.; Feng. T.; Li, T. Investigation of the rheological behavior of activated sludge in response to $\mathrm{CeO}_{2}$ nanoparticles and potential mechanism. Environ. Sci. Pollut. Res 2018, 25, 29725-29733.

(3) Li, L.L.; Tong, Z.H.; Fang, C.Y.; Chu, J.; Yu, H.Q. Response of anaerobic granular sludge to single-wall carbon nanotube exposure. Water Res 2015, 70, 1-8.

(4) Wang, X.; Zhao, Y.; Yuan, B.; Wang, Z.; Li, X.; Ren, Y. Comparison of biofouling mechanisms between cellulose triacetate (CTA) and thin-film composite (TFC) polyamide forward osmosis membranes in osmotic membrane bioreactors. Bioresour. Technol 2016, 202, 50-58.

(5) Adav, S.S.; Lin, J.C.T.; Yang. Z., Whiteley. C.G.; Lee, D.J.; Peng, X.F.; Zhang, Z.P. Stereological assessment of extracellular polymeric substances, exo-enzymes, and specific bacterial strains in bioaggregates using fluorescence experiments. Biotechnol. Adv 2010, 28(2), 255-280.

(6) Jibril, S.; Sirat, H.M.; Basar, N. Bioassay-Guided Isolation of Antioxidants and $\alpha$-Glucosidase Inhibitors from the Root of Cassia sieberiana D.C. (Fabaceae). Rec. Nat. Prod 2017, 11(4), 406-410.

(7) Munyaka, P.M.; Khafipour, A.; Wang, H.; Eissa, N.; Khafipour, E.; Ghia, J.E. Antepartum antibiotic treatment increases offspring susceptibility to experimental colitis: a role of the gut microbiota. Plos One 2015, 10(11), S708-711.

(8) Edgar, R.C. UPARSE: highly accurate OTU sequences from microbial amplicon reads. Nat. Methods 2013, 10(10), 996-998.

(9) Cole J. R.; Wang, Q.; Cardenas, E.; Fish, J.; Chai, B.; Farris, R.J.; et al. The ribosomal database project: improved alignments and new tools for rRNA analysis. Nucleic Acids Res 2009, 37(suppl_1), 141-145.

(10) Wang, Y.; Sheng, H.F.; He, Y.; Wu, J. Y.; Jiang, Y. X.;Tam, F.Y. Comparison of the levels of bacterial diversity in freshwater, intertidal wetland, and marine sediments by using millions of illumina tags. Appl. Environ. Microbiol 2012, 78(23), 8264-8271.

(11) Jiang, X.T.; Peng, X.; Deng, G.H.; Sheng, H.F.; Wang, Y.; Zhou, H.W.;Tam, N.F.Y. Illumina sequencing of 16S rRNA tag revealed spatial variations of bacterial communities in a mangrove wetland. Microb. Ecol 2013, 66(1), 96-104.

(12) Jami, E.; Israel, A.; Kotser, A.; Mizrahi, I. Exploring the bovine rumen bacterial community from birth to adulthood. ISME J 2013, 7(6), 1069-1079.

(13) Chevion, S.; Chevion, M.; Chock, P.B.; Beecher, G.R. Antioxidant capacity of edible plants: extraction protocol and direct evaluation by cyclic voltammetry. J. Med. Food 1999, 2(1), 1-10.

(14) Hoyos-Arbeláez, J.; Vázquez, M.; Contreras-Calderón, J. Electrochemical methods as a tool for determining the antioxidant capacity of food and beverages: A review. Food Chem 2016, 221, 1371-1381.

(15) Kilmartin, P.A.; Hsu, C.F. Characterisation of polyphenols in green, oolong, and black teas, and in coffee, using cyclic voltammetry. Food Chem 2003, 82(4), 501-512.

(16) Kilmartin, P.A. Electrochemical detection of natural antioxidants: principles and protocols. Antioxid. Redox Signaling 2001, 3(6), 941-955.

(17) Chen, B.Y.; Ma, C.M.; Liao, J.H.; Hsu, A.W.; Tsai, P.W.; Wu, C.C.; Hsueh, C.C. Feasibility study on biostimulation of electron transfer characteristics by edible herbs-extracts. J. Taiwan Inst. Chem. Eng 2017, 79, 125-133.

(18) Ayed, L., Chaieb, K., Cheref, A., Bakhrouf, A. Biodegradation and decolorization of triphenylmethane dyes 
by Staphylococcus epidermidis. Desalination 2010, 260(3), 137-146.

(19) Wu, H.J.; Lei, Y.L.; Zhu, R.; Zhao, M.J.; Lu, J.Y.; Xiao, D.; Jiao, C.; Zhang. Z.Q; Shen, G.H.; Li, S.S. Preparation and characterization of bioactive edible packaging films based on pomelo peel flours incorporating tea polyphenol. Food Hydrocolloids 2019, 90, 41-49. 